\title{
Computer assisted determination of acetabular cup orientation using 2D-3D image registration
}

\author{
Guoyan Zheng • Xuan Zhang
}

Received: 12 November 2009 / Accepted: 3 February 2010 / Published online: 24 February 2010

(C) CARS 2010

\begin{abstract}
Background 2D-3D image-based registration methods have been developed to measure acetabular cup orientation after total hip arthroplasty (THA). These methods require registration of both the prosthesis and the CT images to 2D radiographs and compute implant position with respect to a reference. The application of these methods is limited in clinical practice due to two limitations: (1) the requirement of a computer-aided design (CAD) model of the prosthesis, which may be unavailable due to the proprietary concerns of the manufacturer, and (2) the requirement of either multiple radiographs or radiograph-specific calibration, usually unavailable for retrospective studies. In this paper, we propose a new method to address these limitations.

Methods A new formulation for determination of postoperative cup orientation, which couples a radiographic measurement with 2D-3D image matching, was developed. In our formulation, the radiographic measurement can be obtained with known methods so that the challenge lies in the 2D-3D image matching. To solve this problem, a hybrid 2D-3D registration scheme combining a landmark-to-ray 2D-3D alignment with a robust intensity-based 2D-3D registration was used. The hybrid 2D-3D registration scheme allows computing both the post-operative cup orientation with respect to an anatomical reference and the pelvic tilt and rotation with respect to the X-ray imaging table/plate. The method was validated using $2 \mathrm{D}$ adult cadaver hips.
\end{abstract}

Part of the contents of this paper were presented in MICCAI 2007, which was held in Brisbane, Australia, from October 29 to November $2,2007$.

\section{G. Zheng $(\varangle) \cdot X$. Zhang}

Institute for Surgical Technology and Biomechanics,

University of Bern, Stauffacherstrasse 78, 3014 Bern, Switzerland

e-mail: guoyan.zheng@ieee.org
Results Using the hybrid 2D-3D registration scheme, our method showed a mean accuracy of $1.0 \pm 0.7^{\circ}$ (range from $0.1^{\circ}$ to $2.0^{\circ}$ ) for inclination and $1.7 \pm 1.2^{\circ}$ (range from $0.0^{\circ}$ to $3.9^{\circ}$ ) for anteversion, taking the measurements from postoperative CT images as ground truths.

Conclusions Our new solution formulation and the hybrid 2D-3D registration scheme facilitate estimation of postoperative cup orientation and measurement of pelvic tilt and rotation.

Keywords Post-operative cup orientation . $\mathrm{X}$-ray radiograph $\cdot 2 \mathrm{D}-3 \mathrm{D}$ registration .

Total hip arthroplasty $\cdot$ Pelvic tilt

\section{Introduction}

Total hip arthroplasty (THA) has high social-economic impact [1]. Proper component positioning is essential for THA in regard to both the short-term complications and the long-term survival results [2-7]. Previous studies demonstrate that the higher rates of pelvis osteolysis, the severity of the polyethylene wear, and the component migration have all been well associated with the mal-positioning of the acetabular component, and surgical experience indicates that the mal-orientation of the acetabular component in terms of anteversion and inclination is the major cause of dislocation [2-7]. It is thus very important to develop an accurate method to measure the acetabular cup orientation for a reasonable follow-up after THA.

Two-dimensional (2D) anteroposterior (AP) pelvic radiograph is the standard imaging means for measuring the postoperative cup orientation [8-13]. Although it has an inferior 
accuracy in comparison with three-dimensional (3D) techniques based on computed tomography [8-10,13-15], it is used routinely because of its simplicity, availability, and minimal expense associated with its acquisition. While plain pelvic radiographs are easily obtained, measurement of component orientation on plain radiograph is highly inaccurate largely due to the individual pelvis orientation with respect to $\mathrm{X}$-ray plate during radiograph acquisition [8-10,13-15]. For example, the increased pelvic tilt will result in significant decreases in apparent prosthetic cup anteversion and vice versa [16]. Improved methods of measuring component position post-operatively are essential for the accuracy of studies correlating acetabular cup position to osteolysis, wear, and instability, for evaluation of surgical technique and treatment methods, and for our ability to determine optimal range of the component positioning.

2D-3D image registration methods [17-20] have been developed to estimate the post-operative cup orientation with respect to an anatomical reference extracted from a CT study of the patient, which is a plane called the anterior pelvic plane (APP) defined by the anterior superior iliac spines (ASIS) and the pubic tubercles [21-23]. In such methods, both the computer-aided design (CAD) model of the prosthesis and the $\mathrm{CT}$ scan are first registered to the $\mathrm{X}$-ray radiographs, which then allows computing the position of the implant with respect to the APP extracted from the CT images. Although early encouraging results reported in several studies [18-20], the application of these methods is limited in clinical practice due to two limitations: (1) the requirement of a CAD model of the prosthesis [18-20], which may be unavailable due to the proprietary concerns of the manufacturer; and (2) the requirement for either multiple radiographs $[17,20]$ or a radiograph-specific calibration $[18,19]$, usually unavailable for retrospective studies.

To address these limitations, this paper proposes a new formulation for determination of post-operative cup orientation, which couples a radiographic measurement with 2D-3D image matching. In our formulation, the radiographic measurement can be obtained with known methods so the challenge lies in the 2D-3D image matching. To solve this problem, this paper proposes a hybrid 2D-3D registration scheme combining a landmark-to-ray 2D-3D registration with a robust intensity-based 2D-3D registration. Based on this new formulation, we developed an object-oriented program and conducted a preliminary evaluation [24]. Accuracy factors in the determination of post-operative cup orientation using the developed program was also assessed using experiments conducted on one cadaver pelvis [25]. In this paper, we focus on describing the details of the hybrid 2D-3D registration scheme and on evaluating the influence of different registration strategies on the determination accuracy by performing a comprehensive study on ten cadavers (20 hips).

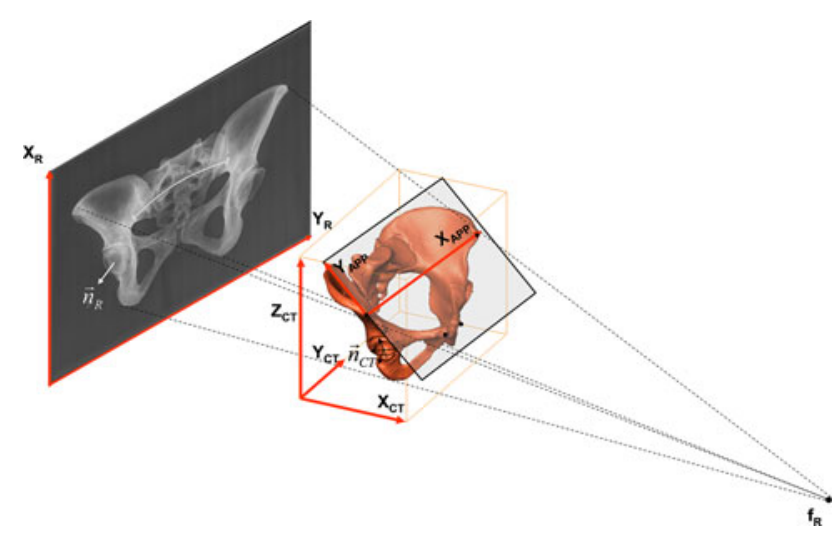

Fig. 1 Schematic illustration of using 2D-3D registration to determine the post-operative cup orientation from single X-ray radiograph

\section{Materials and methods}

The new problem formulation

Unlike previous works [17-20], we formulate the determination of post-operative cup orientation as a process coupling a radiographic measurement with 2D-3D image matching, which can be expressed by the equation as follows (see Fig. 1 for details),

$\vec{n}_{\mathrm{APP}}=T_{\mathrm{APP}}^{\mathrm{CT}} \cdot T_{\mathrm{CT}}^{\mathrm{R}} \cdot \vec{n}_{\mathrm{R}}$

where $\vec{n}_{\mathrm{R}}$ means the orientation of the acetabular cup measured in the $\mathrm{X}$-ray radiograph coordinate system; $T_{\mathrm{CT}}^{\mathrm{R}}$ is the rigid transformation between the $\mathrm{X}$-ray radiograph coordinate system and the CT data coordinate system, and $T_{\mathrm{APP}}^{\mathrm{CT}}$ is the transformation between the CT data coordinate system and a coordinate system defined on APP (see Fig. 1 for details). In order to know the acetabular cup orientation with respect to the APP that is extracted from the CT data, Eq. (1) tells us that one can first compute the acetabular cup orientation with respect to the X-ray radiograph coordinate system and then transform it to the coordinate system defined on the APP through a transformation chain. The advantages of such a problem formulation include (a) the independence of a specific CAD model of the acetabular cup, and (b) the elimination of a radiograph-specific calibration. The only information that we assume to know about the post-operative $\mathrm{X}$-ray radiograph is the image scale (pixel/ $\mathrm{mm}$ ) and the distance from the focal point to the imaging plane or to the film. As long as the radiograph is acquired in a standardized way, which is performed in a clinical routine [12], they can be directly retrieved from the DICOM ${ }^{1}$ header of the image file, if the X-ray image is stored in DICOM format (this is the

\footnotetext{
$\overline{1}$ DICOM means Digital Imaging and Communications in Medicine, which is a standard for handling, storing, printing, and transmitting information in medical imaging. See more details in http://dicom.nema. org/.
} 
way how those two parameters are obtained for all experiments reported in this paper), or can be estimated by performing one-time calibration using a calibration object of known size [26].

There already exist methods for computing $\vec{n}_{\mathrm{R}}$ and $T_{\mathrm{APP}}^{\mathrm{CT}}$ in the literature. In this study, we use the method introduced in [11] to find $\vec{n}_{\mathrm{R}}$ and the method published in [22] to find $T_{\mathrm{APP}}^{\mathrm{CT}}$. The challenge lies in the computation of $T_{\mathrm{CT}}^{\mathrm{R}}$, which is solved by a hybrid $2 \mathrm{D}-3 \mathrm{D}$ registration scheme combining an iterative landmark-to-ray registration with a 2D-3D intensitybased registration (see Sect. "Hybrid 2D-3D registration"). Our 2D-3D registration scheme allows one not only computing the post-operative cup orientation but also computing the pelvic tilt and rotation with respect to the X-ray imaging table/plate.

Our acetabular cup orientation and pelvic tilt and rotation computation protocol is implemented as an object-oriented program called "HipMatch" and consists of following five steps [24]: (1) CT data processing; (2) CT landmark extraction; (3) X-ray landmark extraction; (4) hybrid 2D-3D registration; and (5) acetabular cup orientation and pelvic tilt and rotation computation. For details about each step in our protocol, we refer to our previous work [24]. In the following, we focus on the establishment of the projection geometry of the input X-ray radiograph and on the details about the hybrid 2D-3D registration scheme. Both the establishment of the projection geometry of the X-ray image and the hybrid 2D-3D registration of the X-ray image to the $\mathrm{CT}$ data depend on two sets of anatomical landmarks that are extracted from the CT data and the X-ray radiograph, respectively. The set of 3D anatomical landmarks extracted from the CT data (see Fig. 2a for details) include left and right acetabular centers, the pubic symphysis, and the middle of the sacrococcygeal joint, while their corresponding 2D anatomical landmarks extracted from the X-ray image (see Fig. 2b for details) are left and right acetabular centers, the center of the upper border of the symphysis, and the middle of the sacrococcygeal joint. For details about how to use the "HipMatch" program to interactively extract those anatomical landmarks from the $\mathrm{CT}$ data and from the X-ray image, we refer to our previous work [24].

Establishment of projection geometry of the X-ray radiograph

The projection geometry of the X-ray image is established using the four 2D anatomical landmarks interactively extracted from the X-ray image as follows (see Fig. 3 for details). The intersection between the line connecting the middle of the sacrococcygeal joint and the center of the upper border of the symphysis and the line connecting the acetabular centers is assumed to be the cone-beam projection center and is taken as the coordinate origin. The central projection
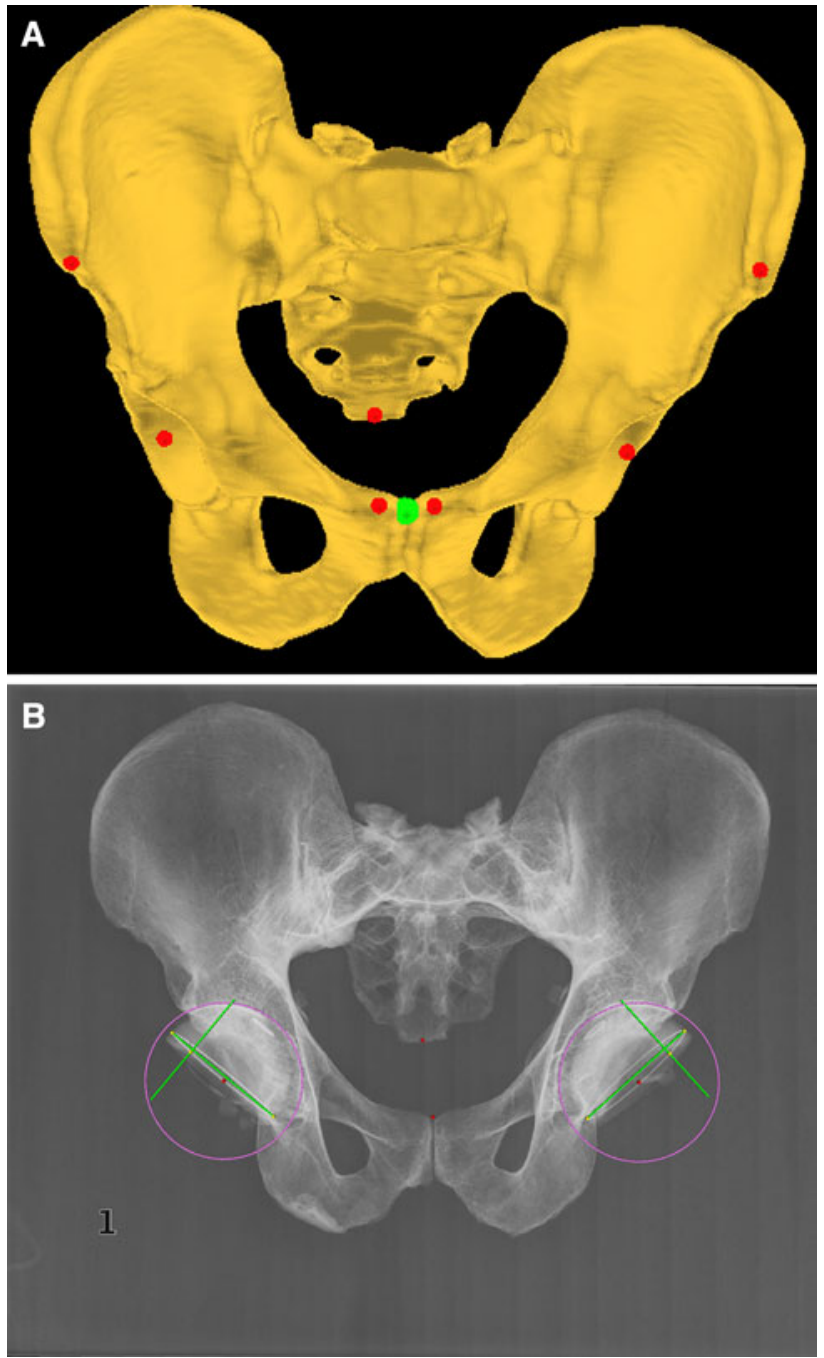

Fig. 2 Anatomical landmarks extracted from the CT data and the X-ray radiograph. (a) Landmarks extracted from the CT data; and (b) Landmarks extracted from the X-ray radiographs

line is perpendicular to the radiograph plane, and its opposite direction is regarded as the $\mathrm{Z}$-axis of the $\mathrm{X}$-ray local coordinate system.

\section{Hybrid 2D-3D registration}

Using those 3D landmarks picked from the $\mathrm{CT}$ data and their corresponding 2D landmarks picked from the radiograph, a landmark-to-ray 2D-3D registration is first performed. Such a problem is known as the perspective-n-points problem in computer vision community, and both analytic solutions and iterative solutions exist before [27]. To avoid ambiguity, we adopted an iterative strategy [28]. The estimated rigid transformation is then treated as the starting value for the next step, i.e., the intensity-based 2D-3D image registration. In this study, we used a spline-based multi-resolution 2D-3D image registration algorithm [29] incorporating a roust similarity 
Fig. 3 Schematic view of the $\mathrm{X}$-ray coordinate system construction and the cone-beam projection model establishment (see text for details)

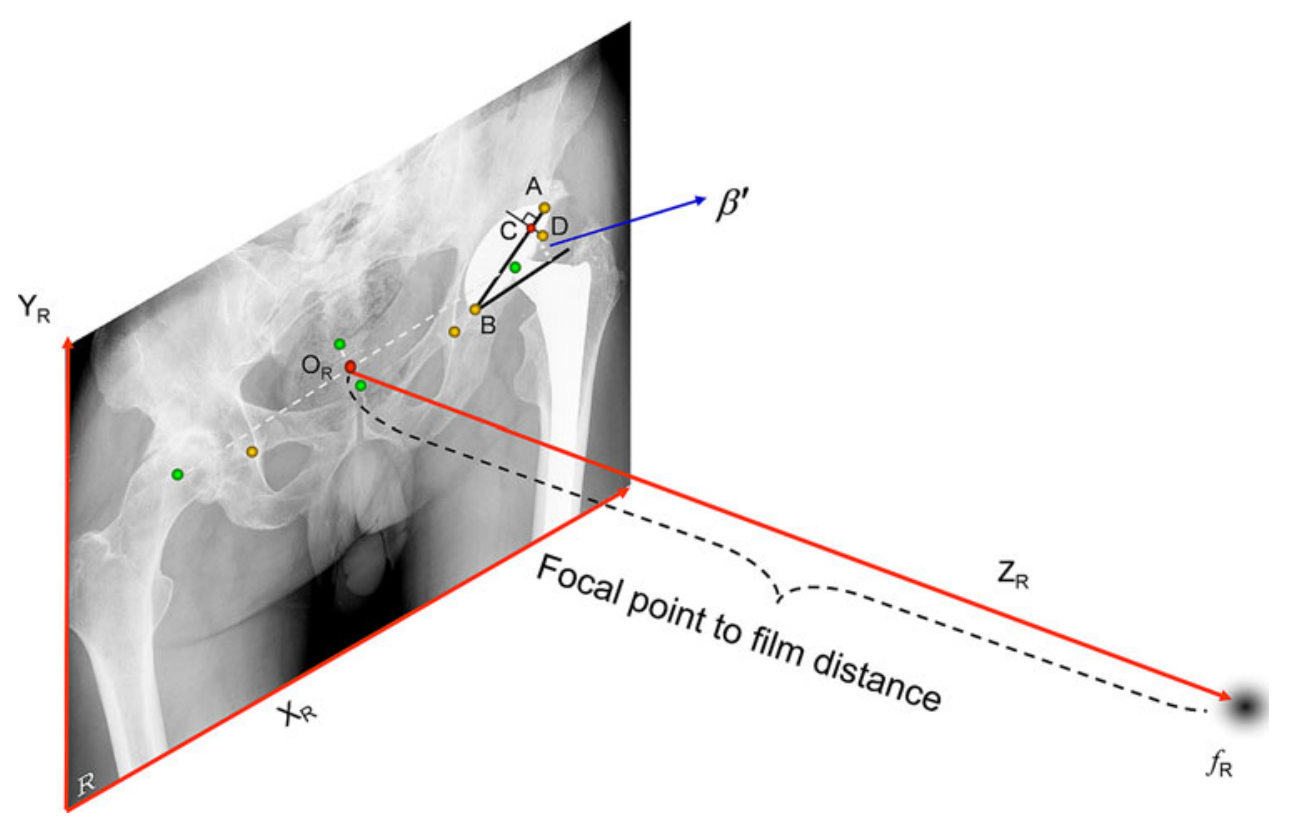

measure that is derived from information and Markov random field theories [30], allowing for effective incorporation of spatial information into the intensity-based 2D-3D image registration. In the following, the details about both steps are given.

\section{Landmark-to-ray $2 D-3 D$ registration}

Let us denote those landmarks defined in CT volume, i.e., the left and right acetabular centers, the pubic symphysis, and the middle of the sacrococcygeal joint, as $v_{\mathrm{CT}}^{1}, v_{\mathrm{CT}}^{2}, v_{\mathrm{CT}}^{3}$ and $v_{\mathrm{CT}}^{4}$, respectively; and their corresponding landmarks interactively picked from the radiograph as $v_{\mathrm{X} \text {-ray }}^{1}, v_{\mathrm{X} \text {-ray }}^{2}, v_{\mathrm{X} \text {-ray }}^{3}$ and $v_{\mathrm{X} \text {-ray }}^{4}$, respectively. And for each X-ray landmark, we can calculate the length between $v_{\mathrm{CT}}^{1}$ and $v_{\mathrm{CT}}^{2}$ and denote it as $l_{\mathrm{CT}}^{1,2}$, and the shortest distance from $v_{\mathrm{CT}}^{3}$ (or $v_{\mathrm{CT}}^{4}$, if the upper border of the symphysis on the radiograph is occluded) to line $v_{\mathrm{CT}}^{1} v_{\mathrm{CT}}^{2}$ and denote it as $l_{\mathrm{CT}}^{3,1-2}$ (or $l_{\mathrm{CT}}^{4,1-2}$ ). Using the known image scale, we also calculate the length $l_{\mathrm{X} \text {-ray }}^{1,2}$ between $v_{\mathrm{X} \text {-ray }}^{1}$ and $v_{\mathrm{X} \text {-ray }}^{2}$. Then, we do:

Initialization: In this step, we assume that the line connecting the acetabular centers is parallel to the AP pelvic radiograph plane. Using this assumption and the correspondences between the landmarks defined in the CT volume and those picked from the radiograph, we can first compute two points $\bar{v}_{\mathrm{X} \text {-ray }}^{1}$ and $\bar{v}_{\mathrm{X} \text {-ray }}^{2}$ on the projection rays of $v_{\mathrm{X} \text {-ray }}^{1}$ and $v_{\mathrm{X} \text {-ray }}^{2}$, respectively, which satisfy:

$$
\begin{gathered}
\bar{v}_{\mathrm{X} \text {-ray }}^{1} \bar{v}_{\mathrm{X} \text {-ray }}^{1} / / v_{\mathrm{X} \text {-ray }}^{1} v_{\mathrm{X} \text {-ray }}^{1} ; \quad \text { and } \\
\left|\bar{v}_{\mathrm{X} \text {-ray }}^{1}-\bar{v}_{\mathrm{X} \text {-ray }}^{2}\right|=l_{\mathrm{CT}}^{1,2}
\end{gathered}
$$

where "//" is a parallel symbol and "|.|" means to compute the distance of two points.

We then find a point $\bar{v}_{\mathrm{X} \text {-ray }}^{3}$ on the projection ray of $v_{\mathrm{X} \text {-ray }}^{3}$ whose distance to the line $\bar{v}_{\mathrm{X} \text {-ray }}^{1} \bar{v}_{\mathrm{X} \text {-ray }}^{1}$ is equal to $l_{\mathrm{CT}}^{3,1-2}$. A paired-point matching [31] based on $\left\{v_{\mathrm{CT}}^{i} ; i=1,2,3\right\}$ and $\left\{\bar{v}_{\mathrm{X} \text {-ray }}^{i} ; i=1,2,3\right\}$ is performed to calculate an initial rigid transformation $\tilde{T}_{\mathrm{CT}}^{\mathrm{X} \text {-ray }}$ (see Fig. 4 a for details). From now on, we assume that all information defined in the CT data coordinate frame has been transformed into the radiograph coordinate frame using $\tilde{T}_{\mathrm{CT}}^{\mathrm{X} \text {-ray }}$. We denote the transformed CT landmarks as $\left\{\tilde{v}_{\mathrm{CT}}^{i}\right\}$.

Iteration. The following steps are iteratively executed until convergence:

1. For a point $\tilde{v}_{\mathrm{CT}}^{i}$, we find a point on the corresponding projection ray of $v_{\mathrm{X} \text {-ray }}^{i}$ which has the shortest distance to the point $\tilde{v}_{\mathrm{CT}}^{i}$ and denote it as $\tilde{v}_{\mathrm{X} \text {-ray }}^{i}$ (see Fig. $4 \mathrm{~b}$ for details). We then performed a paired-point matching using the extracted point pairs to compute a rigid transformation $\Delta \tilde{T}_{\mathrm{CT}}^{\mathrm{X}-\mathrm{ray}}$.

2. We update the CT data coordinate frame using $\Delta \tilde{T}_{\mathrm{CT}}^{\mathrm{X} \text {-ray }}$.

\section{Intensity-based $2 D-3 D$ registration}

Without using fiducial markers, the iterative landmark-to-ray 2D-3D registration cannot fulfill the accuracy requirement of our application and is complemented by an intensity-based $2 \mathrm{D}-3 \mathrm{D}$ registration. The challenge here is the big area occlusion, which may be caused by the projection of a gonadal shielding, which is an effective way of reducing radiation dose to patient's reproduction organs, or the projections of 
Fig. 4 Schematic view of the iterative landmark-to-ray 2D-3D registration.

(a) Schematic view of the initialization; and (b) schematic view of finding $3 \mathrm{D}$ point pairs
A

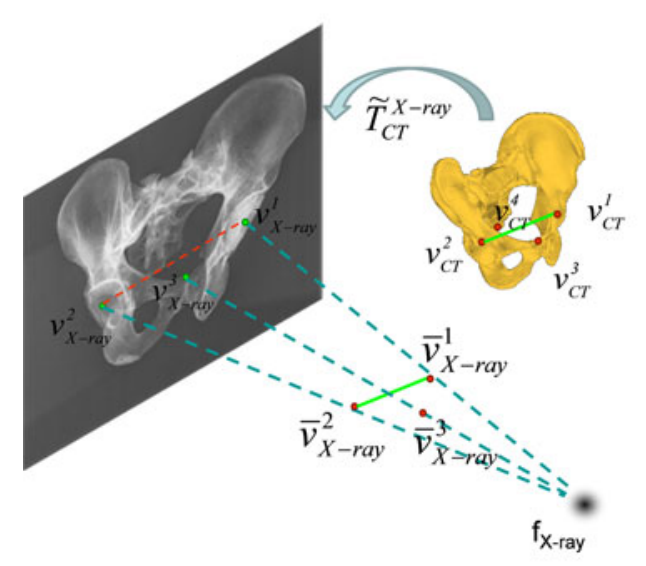

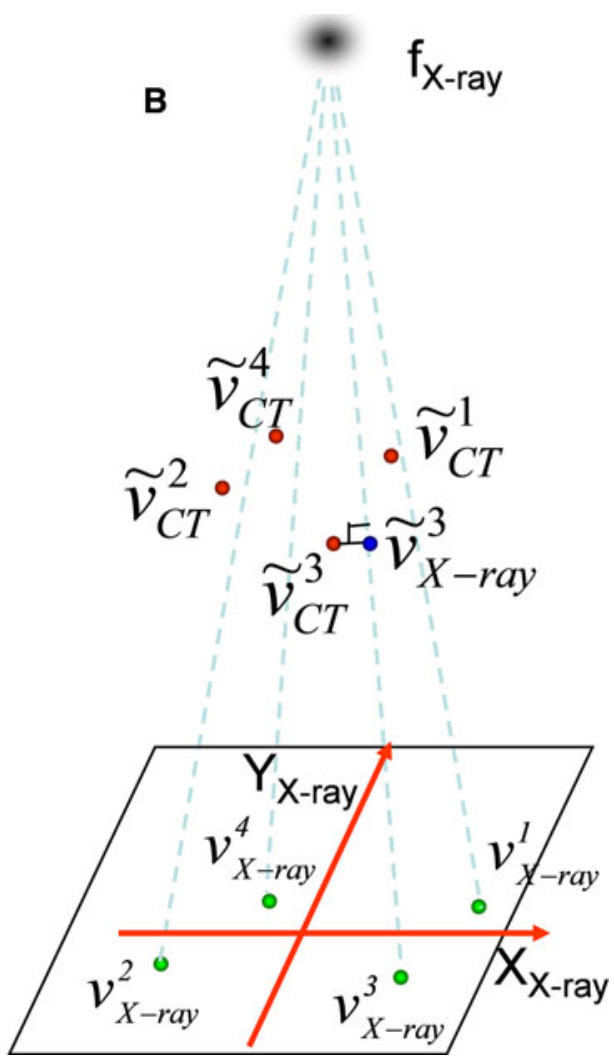

the implanted prostheses. Such occlusion creates large differences between the X-ray radiograph and the digitally reconstructed radiographs (DRR) obtained from the CT data by simulating X-ray projection given the current estimation of the pose of the CT data with respect to the radiograph coordinate frame and contains very little useful information to aid registration.

Let us denote $L=\{(i, j): 1 \leq i \leq I, 1 \leq j \leq J\}$, an $I \times J$ integer lattice, as the pixel sites of the X-ray radiograph, and the image value at pixel site $(i, j)$ of the X-ray radiograph as $I_{\mathrm{X} \text {-ray }}(i, j)$. Similarly, we denote the image value of the DRR at pixel site $(i, j)$ as $I_{\mathrm{DRR}}(i, j)$. Our intensity-based 2D-3D registration scheme is based on a recently introduced spline-based multi-resolution 2D-3D registration scheme [29] but with a different similarity measure. We use a similarity measure that is derived from information and Markov random field theories [30]. It allows us to effectively incorporate spatial information and has following form,

$S=\sum_{i, j}^{I, J} d_{i, j}^{2}+\sum_{i, j}^{I, J} \frac{1}{\operatorname{card}\left(N_{i, j}^{r}\right)-1} \sum_{\left(i^{\prime}, j^{\prime}\right) \in N_{i, j}^{r}}\left(d_{i, j}-d_{i^{\prime}, j^{\prime}}\right)^{2}$

where $N_{i, j}^{r}$ is a neighborhood defined by,

$N_{i, j}^{r}=\left\{\left(i^{\prime}, j^{\prime}\right)\left|\left(i^{\prime}, j^{\prime}\right) \in L,\right|\left(i^{\prime}, j^{\prime}\right)-(i, j) \mid \leq r\right\}$ and $r$ is a positive integer that determines the size of the neighborhood system; $\operatorname{card}\left(N_{i, j}^{r}\right)$ means to compute the number of the pixels in the local region $N_{i, j}^{r} ; d_{i, j}$ is the local normalization-based difference image value at pixel site $(i, j)$ and is computed by,

$$
\left\{\begin{array}{l}
d_{i, j}=\bar{I}_{\mathrm{X}-\text { ray }}(i, j)-\bar{I}_{\mathrm{DRR}}(i, j) \\
\bar{I}_{\mathrm{X} \text {-ray }}(i, j)=\frac{I_{\mathrm{X}-\text { ray }}(i, j)-m_{\mathrm{X}-\text { ray }}\left(N_{i, j}^{r}\right)}{\sigma_{\mathrm{X}-\text { ray }}\left(N_{i, j}^{r}\right)} \\
\bar{I}_{\mathrm{DRR}}(i, j)=\frac{I_{\mathrm{DRR}}(i, j)-m_{\mathrm{DRR}}\left(N_{i, j}^{r}\right)}{\sigma_{\mathrm{DRR}}\left(N_{i, j}^{r}\right)}
\end{array}\right.
$$

where $m_{\mathrm{X} \text {-ray }}\left(N_{i, j}^{r}\right), \sigma_{\mathrm{X} \text {-ray }}\left(N_{i, j}^{r}\right)$ and $m_{\mathrm{DRR}}\left(N_{i, j}^{r}\right), \sigma_{\mathrm{DRR}}$ $\left(N_{i, j}^{r}\right)$ are the mean value and the standard deviation calculated from the intensity values of all sites in the local region $N_{i, j}^{r}$ of the X-ray radiograph and of the associated DRR, respectively.

To accelerate the registration process, we used the cubicsplines data model described in [29] to compute the multiresolution data pyramids for both the CT data and the X-ray image, the DRR, as well as the gradient and the Hessian of the similarity measure. The registration is then performed from the coarsest resolution until the finest one. And to improve the capture range, we used two different sizes of neighborhood systems: $r=15$ and $r=3$. Starting from the rigid transformation obtained by the iterative landmark-to-ray registration, the similarity measure with the bigger neighborhood system is first minimized via a Levenberg-Marquardt non- 

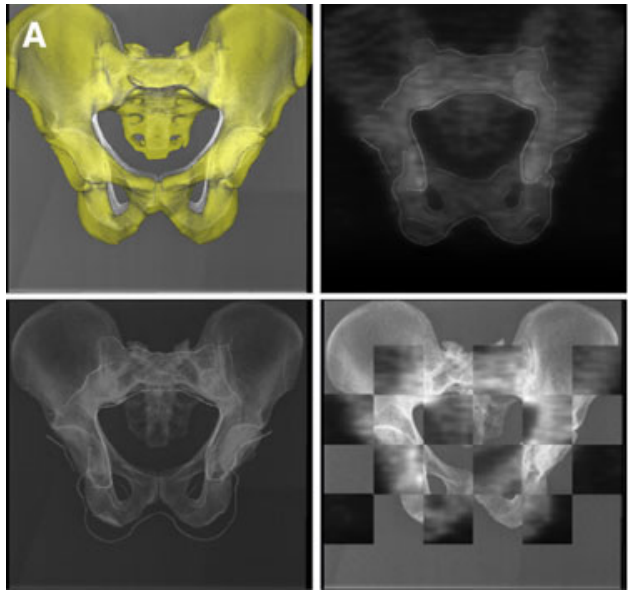

Fig. 5 Screenshots of applying the hybrid 2D-3D registration scheme to a cadaver dataset. (a) The result after the landmark-to-ray 2D-3D registration is applied; and (b) the result after the intensity-based 2D-3D registration is applied. In both images, the top left window shows the superposition of the segmented CT surface model on the

linear least-squares optimizer [29]. The estimated rigid transformation is then treated as starting value for optimizing the similarity measure with the smaller neighborhood system.

Figure 5 shows screenshots of applying the hybrid 2D-3D registration scheme to a cadaver data set. The result of the landmark-to-ray registration is shown by Fig. 5a. Starting from the initial transformation estimated from the landmarkto-ray registration, the intensity-based $2 \mathrm{D}-3 \mathrm{D}$ registration further optimizes the similarity measure between the X-ray image and the DRR, and the final result is shown by Fig. 5b.

\section{Experiments}

We performed an experiment on ten cadavers (20 hips) to validate the efficacy of the present method. Each cadaver was bilaterally implanted with prosthetic polyethylene liner (Charles F. Thackray, Leeds, UK). The liners were marked with a circular metal wire. A pre-implantation CT scan, a post-implantation CT scan, and a post-implantation X-ray radiograph were acquired for each cadaver. All radiographs were taken in a roughly neutral position with the focal point to film distance being $1,200 \mathrm{~mm}$. For each case, to avoid the error introduced by the differences in APP definitions, we decided to use the post-implantation CT scan for both the ground truth extraction and the measurements obtained from the present method. To this end, acetabular cup orientations measured using the present method and by measurement directly from the 2D X-ray images using the method introduced in [11] were compared to the ground truths calculated from the associated CT scan. All measurements are input to Microsoft Excel ${ }^{\circledR} 2007$ (Microsoft Corporation,

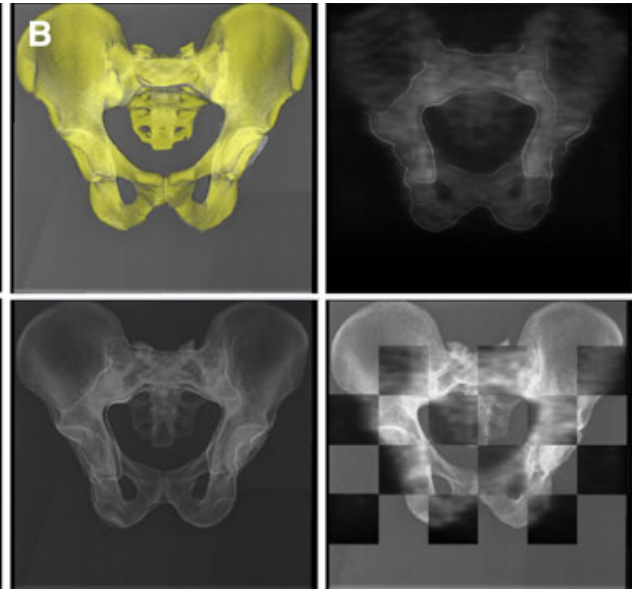

X-ray image; the top right window shows the DRR created from a down-sampled CT data; the bottom left window shows the superposition of the edges extracted from the DRR on the X-ray image; and the bottom right window shows the check board display of the DRR and the X-ray image

Redmond, USA) for statistics. The significant level was chosen as $\alpha=0.01$.

For all experiments, we used an Intel Duo Core 2.4- GHz laptop with 4GB of RAM. All programming was done using Visual C++ 2005 on Windows Vista; additional functionality was implemented using a cross-platform graphic user interface programming framework Qt 3.3.6 (TrollTech, Oslo, Norway).

The ground truth of each case is obtained from the associated post-implantation CT scan as follows. We first extract the APP from the post-implantation CT data by interactively picking four anatomic landmarks using the "HipMatch" program [24]. The four anatomical landmarks for defining the APP are bilateral anterior superior iliac spine and bilateral pubic tubercle, all defined at anterior tips of cortical bone. Their exact location can be fine-tuned interactively through three orthogonal multi-planar reconstruction viewers as implemented in the "HipMatch" program [24]. Furthermore, to estimate the acetabular cup orientation, we need to define the normal to the cup opening plane, which is done by interactively picking another three points from the post-operative CT data. The positions of these three points are again interactively controlled using the three orthogonal multi-planar reconstruction viewers as implemented in the "HipMatch" program (see Fig. 6 for an example). The ultimate goal is to make sure that all these three points fall on a plane that is parallel to the plane of the cup opening. As soon as all landmarks are extracted from the post-operative CT data, we can compute the acetabular cup orientation with respect to the APP. According to Murray [32], acetabular cup anteversion and inclination can be defined in three different ways (see Fig. 7 for a schematic illustration), i.e, radiological 
Fig. 6 Screenshots of the graphical user interface for interactively defining points on the circular metal wire of an implanted cup from the post-implantation CT scan. From these points, we can construct a plane that is parallel to the cup opening plane. See text for details
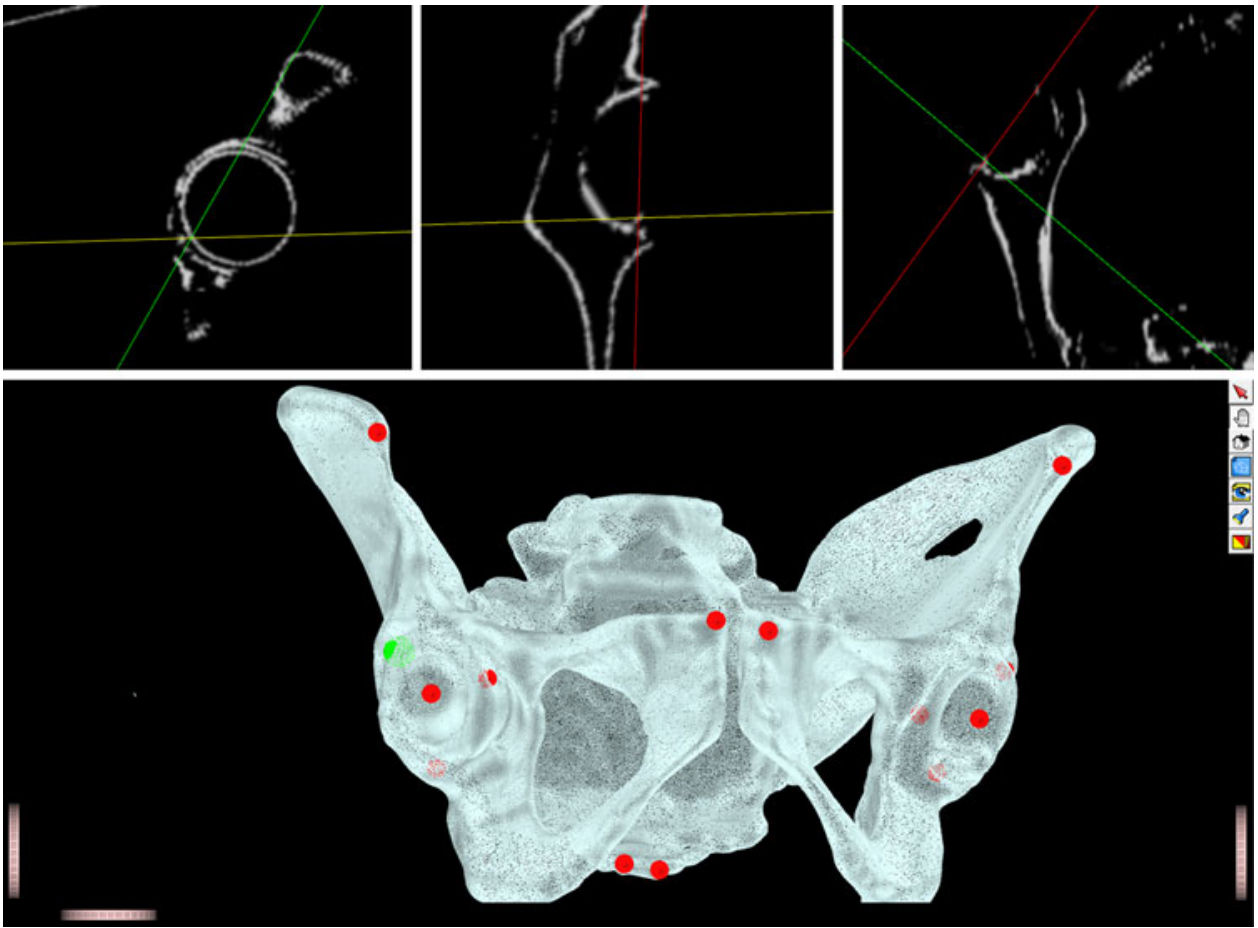

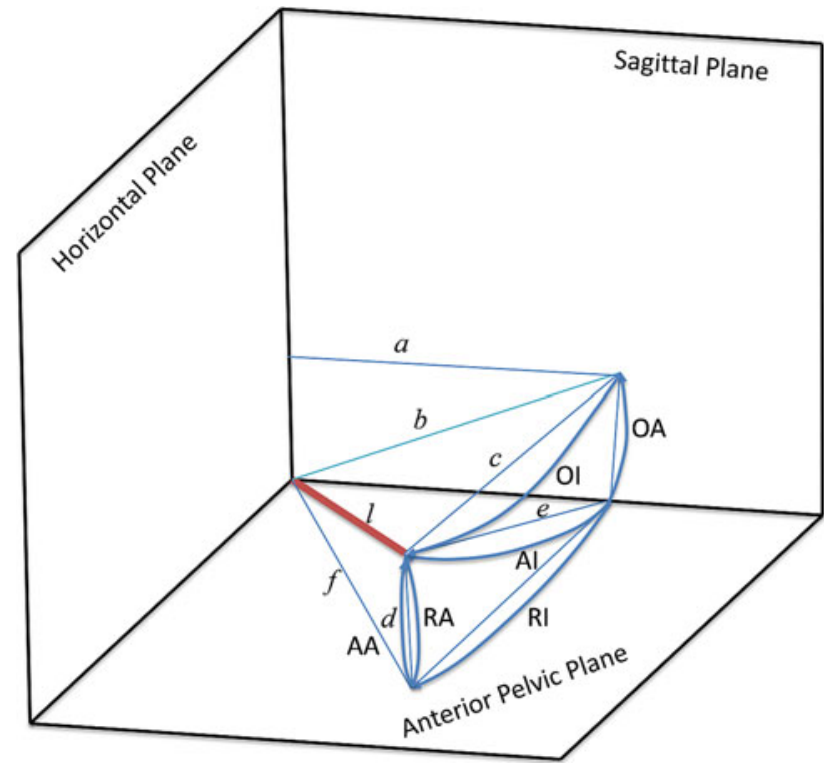

Fig. 7 Schematic view of different definitions of cup anteversion and inclination according to Murray [32]

inclination $(R I)$ and radiological anteversion $(R V)$, anatomical inclination $(A I)$ and anatomical anteversion $(A A)$, and operative inclination $(O I)$ and operative anteversion $(O A)$. In this paper, for both the ground truth measurement and the measurement generated from the present method, we used anatomical anteversion $(A A)$ and radiographic inclination $(R I)$. The reason why these particular angles are chosen is due to their wide usage in most of the commercial nav- igation systems [20,33]. Thus, the radiological anteversion measured from the plain film using the technique described in [11] is converted to the anatomical anteversion using a formula described by Murray [32] for later comparison purpose.

Using these 20 cadaver hips, we evaluated the influences of different registration strategies on the determination accuracy. The purposes of the experiment were twofold. First, we would like to measure the estimation accuracy achieved by each registration step. Second, we would like to know whether the use of the landmark-to-ray 2D-3D registration alone is enough for our task. For each hip, three measurement results were recorded and compared to the associated ground truth: (1) the measurement result based on the conventional plain film technique described in [11], assuming that the APP are parallel to the X-ray imaging table/plate; (2) the measurement result after the landmark-to-ray 2D-3D registration was applied; and (3) the measurement result after the hybrid 2D-3D registration scheme was applied.

Furthermore, the transformation that is obtained from the hybrid 2D-3D registration also allows one to transform the APP from the CT data coordinate system to the X-ray image coordinate system for a computation of the pelvic tilt with respect to the $\mathrm{X}$-ray imaging table/plate. The pelvic tilt angle is defined as the difference between the anatomical coordinate system defined on the APP of the pelvis and the plane of the X-ray imaging table/plate around the pelvic horizontal axis, while the pelvic rotation angle is defined as the difference between the anatomical coordinate system defined on the APP of the pelvis and the plane of the X-ray imaging 


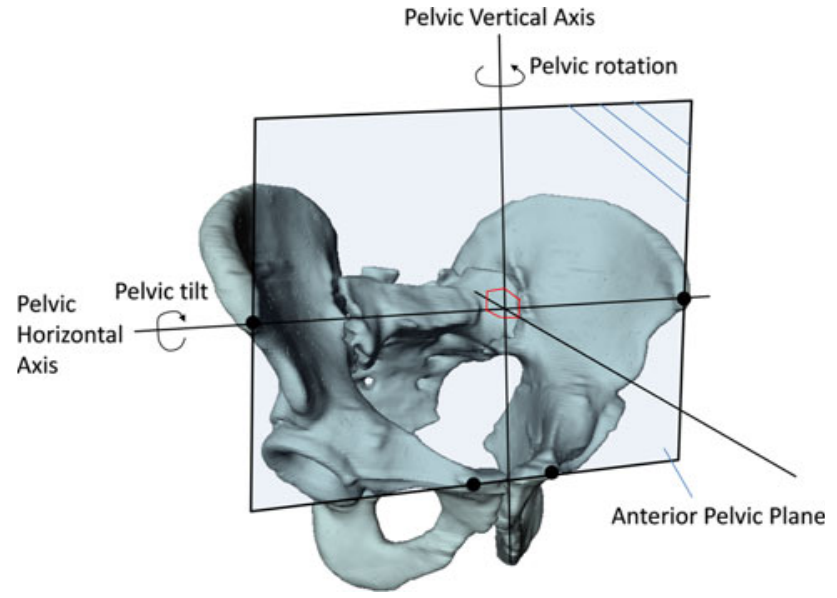

Fig. 8 Schematic view of the definitions of the pelvic tilt and the pelvic rotation

table/plate around the pelvic vertical axis. See Fig. 8 for a schematic illustration of the definitions of these two angles.

\section{Experimental results}

The comparison results between the radiographic measurements and the ground truths are presented in Table 1. A mean absolute difference of $3.2^{\circ} \pm 3.0^{\circ}$ (range from $0.4^{\circ}$ to $8.5^{\circ}$ ) was found for the inclination, and a mean absolute difference of $5.9^{\circ} \pm 3.2^{\circ}$ (range from $0.2^{\circ}$ to $15.2^{\circ}$ ) was found for the anteversion when the radiographic measurements were compared to the ground truth.

The absolute differences between the ground truths and the measurements obtained when only the landmark-to-ray 2D-3D registration was applied and the absolute differences between the ground truths and the measurements obtained when the hybrid 2D-3D registration was applied are presented in Tables 2 and 3, respectively. When only the landmark-to-ray 2D-3D registration was applied, the mean absolute difference for the inclination was changed to $1.3^{\circ} \pm 0.9^{\circ}$ (range from $0.1^{\circ}$ to $4.0^{\circ}$ ) and the mean absolute difference for the anteversion was changed to $4.2^{\circ} \pm 2.5^{\circ}$ (range from $0.5^{\circ}$ to $8.0^{\circ}$ ). Using a paired two-tailed $t$ test, it was found that both improvements on the inclination observation and those on the anteversion observation were not statistically significant (see Table 4 for details). However, when the hybrid 2D-3D registration was applied, the mean absolute difference for the inclination was further decreased to $1.0^{\circ} \pm 0.7^{\circ}$ (range from $0.1^{\circ}$ to $2.0^{\circ}$ ) and the mean absolute difference for the anteversion was further decreased to $1.7^{\circ} \pm 1.2^{\circ}$ (range from $0.0^{\circ}$ to $3.9^{\circ}$ ). The differences between the measurements obtained from the hybrid 2D-3D registration scheme and those obtained from the radiographic calculation were statistically significant for both the inclination observation $(p=0.002<0.01)$ and the anteversion observation ( $p=1.4 \mathrm{E}-05<0.01)$, which demonstrated (1) the efficacy of the present method and (2) that the landmark-to-ray 2D-3D registration alone was not accurate enough for our task. Statistically, the differences between the measurements obtained from applying the hybrid 2D-3D registration scheme and those obtained from applying only the landmark-to-ray 2D-3D registration were significant for anteversion observation $(p=8.8 \mathrm{E}-05<0.01)$ but not for inclination observation $(p=0.04>0.01)$.

The experiment on measuring the pelvic tilt and rotation angles of all ten cadavers showed following results: an average of $3.8^{\circ} \pm 6.5^{\circ}$ (range from $-8.6^{\circ}$ to $10.3^{\circ}$ ) was found for the pelvic tilt angles and an average of $-0.4^{\circ} \pm 1.5^{\circ}$ (range from $-2.9^{\circ}$ to $2.7^{\circ}$ ) was found for the pelvic rotation angles. We also recorded the computation time for each case required by the present method. On average, it took the present method $42.6 \pm 17.2 \mathrm{~s}$ to finish the computation.

\section{Discussions and conclusions}

Accurate assessment of the acetabular cup orientation is important for evaluation of outcome after THA, but the inability to measure acetabular cup orientation accurately
Table 1 Results of comparing the ground truths with the radiographic measurements obtained using the method published in [11] $(n=20)$

\begin{tabular}{lllc}
\hline Angle & Mean $\pm \operatorname{STD}\left(^{\circ}\right)$ & Minimum error $\left(^{\circ}\right)$ & Maximum error $\left(^{\circ}\right)$ \\
\hline Inclination & $3.2 \pm 3.0$ & 0.4 & 8.5 \\
Anteversion & $5.9 \pm 3.2$ & 0.2 & 15.2 \\
\hline
\end{tabular}

Table 2 Results of comparing the ground truths with the measurements after the landmark-to-ray 2D-3D registration was applied $(n=20)$

\begin{tabular}{llll}
\hline Angle & Mean $\pm \operatorname{STD}\left(^{\circ}\right)$ & Minimum error $\left(^{\circ}\right)$ & Maximum error $\left(^{\circ}\right)$ \\
\hline Inclination & $1.3 \pm 0.9$ & 0.1 & 4.0 \\
Anteversion & $4.2 \pm 2.5$ & 0.5 & 8.0 \\
\hline
\end{tabular}


Table 3 Results of comparing of the ground truths with the measurements after the hybrid 2D-3D registration scheme was applied $(n=20)$

\begin{tabular}{llll}
\hline Angle & Mean $\pm \operatorname{STD}\left({ }^{\circ}\right)$ & Minimum error $\left(^{\circ}\right)$ & Maximum error $\left(^{\circ}\right)$ \\
\hline Inclination & $1.0 \pm 0.7$ & 0.1 & 2.0 \\
Anteversion & $1.7 \pm 1.2$ & 0.0 & 3.9 \\
\hline
\end{tabular}

Table 4 The paired two-tailed $t$-test results when the results obtained using different registration strategies were compared to each other

\begin{tabular}{llll}
\hline Comparisons & $\begin{array}{l}\text { Differences between the } \\
\text { ground truth and the } \\
\text { radiograph measurements } \\
\text { versus differences between } \\
\text { the ground truths and the } \\
\text { measurement results when } \\
\text { the landmark-to-ray 2D-3D } \\
\text { registration was applied }\end{array}$ & $\begin{array}{l}\text { Differences between the } \\
\text { ground truth and the } \\
\text { radiograph measurements } \\
\text { versus differences between } \\
\text { the ground truths and the } \\
\text { measurement results when the } \\
\text { hybrid 2D-3D registration } \\
\text { scheme was applied }\end{array}$ & $\begin{array}{l}\text { Differences between the } \\
\text { ground truth and the } \\
\text { measurement results when } \\
\text { the landmark-to-ray 2D-3D } \\
\text { registration was applied } \\
\text { versus differences between } \\
\text { the ground truths and the } \\
\text { measurement results when the } \\
\text { hybrid 2D-3D registration } \\
\text { scheme was applied }\end{array}$ \\
\hline $\begin{array}{l}P \text {-value of inclination observation } \\
P \text {-value of anteversion observation }\end{array}$ & $0.014>0.01$ & $\begin{array}{l}0.002<0.01 \\
1.5 \mathrm{E}-05<0.01\end{array}$ & $0.04>0.01$ \\
\hline
\end{tabular}

limits one's ability to determine optimal cup orientations, to assess new treatment methods of improving acetabular cup orientation in surgery, and to correlate the acetabular cup orientation to osteolysis, wear, and instability. The goal of the present study is to develop and validate a new technique for measuring post-operative cup orientation. Through formulating the determination of post-operative cup orientation as a process of coupling a radiographic measurement with 2D-3D image matching, we addressed limitations in the existing methods [17-20], which prevents those methods from wide usage in clinical routine. The results of the experiments that were conducted on datasets of ten cadavers (20 hips) demonstrated that the present technique was apparently more accurate than the radiographic measurement method. The substantial error range (from $0.2^{\circ}$ to $15.2^{\circ}$ ) as found in measuring anteversion using the radiographic measurement method [11] in this study is coincident with other recently published findings that the radiographic measurement of anteversion is unreliable [8-10,13-15,24,25].

The advantages of the present technique over other methods for post-operative calculation of acetabular cup orientation are apparent. Several studies [8-10,13-15,24,25], including the one presented in this paper, have shown that the radiographic measurement methods, though easy to use, cannot produce accurate results due to the wide variability in individual pelvic orientation relative to X-ray plate during image acquisition. Post-operative CT-based method, which is regarded as the most reliable method for measuring post-operative cup orientation [8-10,13-15,34,35], cannot be used in clinical routine due to the additional expense and radiation doses to the patient associated with its acquisition. As long as there is a CT study of the patient during treatment, the present technique can be used to accurately and reliably measure the post-operative cup orientation and the pelvic tilt and rotation.

There are also significant differences between the present technique and other published methods [17-20] on measuring post-operative cup orientation using 2D-3D image matching. Due to the new formulation, the present technique does not ask for multiple $\mathrm{X}$-ray radiographs as required in $[17,20]$, nor requires a radiography-specific calibration as in $[18,19]$. More importantly, the present technique does not need a CAD model of the prosthesis. An additional matching process of the CAD model of the prosthesis to the projected cup [18-20] is thus unnecessary. The additional step to match the CAD model to the projected cup in the existing work was either performed manually $[18,20]$ or a manual coarse alignment was initially required [19]. This implicates, according to the authors, a certain degree of subjective judgment. The advantages of the present approach for long-term retrospective studies have been demonstrated by the convincing results of a recent validation study [36]. In this study, measurements on blinded and randomized radiographs of 80 cadaver and 327 patient hips were performed. When compared to CT-based measurements, our approach showed a mean accuracy of $0.7^{\circ} \pm 1.7^{\circ}\left(-3.7^{\circ}\right.$ to $\left.4.0^{\circ}\right)$ for inclination and $1.2^{\circ} \pm 2.4^{\circ}\left(-5.3^{\circ}\right.$ to $\left.5.6^{\circ}\right)$ for anteversion in the cadaver trials, and $1.7^{\circ} \pm 1.7^{\circ}\left(-4.6^{\circ}\right.$ to $\left.5.5^{\circ}\right)$ for inclination and $0.9^{\circ} \pm 2.8^{\circ}\left(-5.2^{\circ}\right.$ to $\left.5.7^{\circ}\right)$ for anteversion in the clinical trial [36]. This study also demonstrated that the software consistency and the reproducibility/reliability were very good [36].

It is worth to note that all three ways of acetabular cup orientation measurement as described by Murray [32] can be based on either the (1) anterior pelvic plane or (2) coronal plane. All the cup orientation measurement results reported 
in this paper were compared to the associated ground truths that were measured with respect to the anterior pelvic planes extracted from the post-operative CT scans. As demonstrated by the experimental results, the differences between the radiographic measurements and the ground truths were mainly caused by the tilt angle between the anterior pelvic plane, which was the reference plane that the ground truths were based on, and the X-ray imaging plane/plate, which was the reference plane that the radiographic measurements were based on. It was suggested by Wan et al. [37] that the common measurements used for reporting compute navigation and CT scan values should be the radiographic coronal plane because of the reason that surgeons were familiar with the measurement numbers of the radiographic coronal plane of plain radiographs. However, the reality is that most of the available studies on computer navigation based or CT scan-based measurements are done with respect to the anterior pelvic plane without considering the pelvic tilt [8-10,15,18-22,24, 25,33,34,36], although the influence of the pelvic tilt on anteversion and inclination of the acetabular cup has drawn more and more attentions [37-42]. The present method, which allows for computing the pelvic tilt and rotation with respect to the $\mathrm{X}$-ray imaging plane/plate by performing a 2D-3D registration between a CT scan of the patient and a post-operative X-ray radiograph, can be used as a tool to convert the results measured with respect to the anterior pelvic plane to those measured with respect to the radiographic coronal plane.

While accurate, our method has limitations. The precondition of the implant that can be measured by our method is a circular opening surface of the acetabular cup. Still, due to the fact that the method according to Pradhan et al. [11] is used to calculate the cup version from the plain radiograph, our method is applicable to all type of bearings. The second limitation is that our method in its current form only measures the acetabular cup orientation. Thus, more work needs to be done to support the combined anteversion technique [43]. The third limitation is that our method is currently limited only to those hips that have had a CT study at some point during the treatment. Nevertheless, as demonstrated by our recent work [44], such a constraint can be totally eliminated through a statistically deformable registration of a statistical shape model of the pelvis to the X-ray image.

In summary, we presented a hybrid $2 \mathrm{D}-3 \mathrm{D}$ registration method to estimate the rigid transformation between a CT scan of the patient and a post-operative X-ray radiograph. Such a registration allows one not only to compute the postoperative cup orientation with respect to the patient's individualized anterior pelvic plane but also to compute the pelvic tilt and rotation with respect to the X-ray imaging plane/plate, which is required when one would like to take the influence of the pelvic tilt on anteversion and inclination of the acetabular cup into consideration. Compared to previously introduced methods [17-20], our method has several advantages. Our method does not need a proprietary CAD model of the prosthesis, and our approach does not ask for multiple radiographs, or a radiograph-specific calibration. Thus, we are confident that our method can be used retrospectively to provide evidence-based information for evaluating surgical technologies (e.g., surgical navigation) and implant design.

Acknowledgements This work was partially financed by the Swiss National Science Foundation (SNSF) through the project NCCRCOME. The author would like to thank Dr. Simon Steppacher and Dr. Moritz Tannast for their help in experimental setup. The authors would like to thank the anonymous reviewers for their constructive comments and suggestions.

\section{References}

1. Kiefer H (2007) Differences and opportunities of THA in the USA, Asia and Europe, In: Chang J-D, Billau K (eds) Ceramics in orthopaedics, the proceedings of the 12 th BIOLOX $^{\circledR}$ symposium on bioceramics and alternative bearings in joint arthroplasty. Seoul, Republic of Korea, September 7-8, 2007, pp 3-8, Springer

2. Ali Kahn MA, Brakenbury PH, Reynolds IS (1981) Dislocation following total hip replacement. J Bone Joint Surg-Br 63(2):214218

3. McCollum DE, Gray WJG (1990) Dislocation after total hip arthroplasty. Causes and prevention. Clin Orthop Relat Res 261: $159-170$

4. Bader RJ, Steinhauser E, Willmann G, Gradinger R (2001) The effects of implant position, design and wear on the range of motion after total hip arthroplasty. Hip Int 11(2):80-90

5. Lewinnek GE, Lewis JL, Tarr R, Compere CL, Zimmerman JR (1978) Dislocations after total hip-replacement arthroplasties. J Bone Joint Surg_Am 60(2):217-220

6. Patil S, Bergula A, Chen PC, Colwell CW Jr, D'Lima DD (2003) Polyethylene wear and acetabular component orientation. J Bone Joint Surg [Am] 85:56-63

7. Widmer KH (2007) Containment versus impingement: finding a compromise for cup placement in total hip arthroplasty. Int Orthop 31(Suppl 1):S29-S33

8. Thomas T, Handel M, Herold T, Perlick L, Paetzel C, Grifka J (2006) Position of the acetabular cup-accuracy of radiographic calculation compared to CT-based measurement. Eur J Radiol 58:294-300

9. Marx A, von Knoch M, Pförtner M, Wiese M, Saxler G (2006) Misinterpretation of cup anteversion in total hip arthroplasty using planar radiography. Arch Orthop Trauma Surg 126:487-492

10. Tannast M, Zheng G, Anderegg C, Burckhardt K, Langlotz F, Ganz R, Siebenrock KA (2005) Tilt and rotation correction of acetabular version on pelvic radiographs. Clin Orthop Relat Res 438:182-190

11. Pradhan R (1999) Planar anteversion of the acetabular cup as determined from plain nteroposterior radiographs. J Bone Joint Surg Br 81(B):431-435

12. Della Valle CJ, Kaplan K, Jazrawi A, Ahmed S, Jaffe WL (2004) Primary total hip arthroplasty with a flanged, cemented allpolyethylene acetabular component: evaluation at a minimum of 20 years. J Arthroplasty 19:23-26

13. Arai N, Nakamura S, Matsushita T, Suzuki S (2009) Minimal radiation dose computed tomography for measurement of cup orientation in total hip arthroplasty. J Arthroplasty, in press. doi:10.1016/ j.arth.2009.01.020 
14. Clohisy JC, Carlisle JC, Trousdale R et al (2009) Radiographic evaluation of the hip has limited reliability. Clin Orthop Relat Res 467:666-675

15. Ghelman B, Kepler CK, Lyman S, Della Valle AG (2009) CT outperforms radiography for determination of acetabular cup version after THA. Clin Orthop Relat Res 467:2362-2370

16. Sellers RG, Lyles D, Dorr LD (1988) The effect of pelvic rotation on alpha and theta angles in total hip arthroplasty. Contemp Orthop 17:67-70

17. Larose D, Cassenti L, Jaramaz B, Moody JE, Kanade T, DiGioia AM (2000) Post-operative measurment of acetabular cup position using X-ray/CT registration. In: Proceedings of the 3rd international conference on medical image computing and computer-assisted intervention (MICCAI 2000), LNCS 1935, pp 1104 1113

18. Blendea S, Eckman K, Jaramaz B, Levison TJ, DiGioia AM (2005) Measurements of acetabular cup position and pelvic spatial orientation after total hip arthroplasty using computed tomography/radiography matching. Comput Aided Surg 10:37-43

19. Jaramaz B, Eckman K (2006) 2D/3D registration for measurement of implant alignment after total hip replacement. In: Proceedings of the 9th international conference on medical image computing and computer-assisted intervention (MICCAI 2006), LNCS 4191, pp 653-661

20. Penney GP, Edwards PJ, Hipwell JH, Slomczykowski M, Revie I, Hawkes DJ (2007) Postoperative calculation of acetabular cup position using 2D-3D registration. IEEE T Biomed Eng 54:3421348

21. Tannast M, Langlotz U, Siebenrock KA, Wiese KM, Bernsmann K, Langlotz F (2005) Anatomical referencing of cup orientation in total hip arthroplasty. Clin Orthop Relat Res 436:144-150

22. Jaramaz B, DiGioia AM, Blackwell M, Nikou C (1998) Computer assisted measurement of cup placement in total hip replacement. Clin Orthop Relat Res 354:70-81

23. Langlotz U, Grützner PA, Bernsmann K, Kowal J, Tannast M, Caversaccio M, Nolte L-P (2007) Accuracy considerations in navigated cup placement for THR. Proc Inst Mech Eng [H] 221:739753

24. Zheng G, Zhang X, Steppacher S, Murphy SB, Siebenrock KA, Tannast M (2009) HipMatch: an object-oriented cross-platform program for accurate determination of cup orientation using 2D-3D registration of single standard X-ray radiograph and CT volume. Comput Methods Program Biomed 95:236-248

25. Zheng G (2009) Assessing the accuracy factors in the determination of postoperative acetabular cup orientation using hybrid 2D-3D registration. J Digit Imaging, in press. doi:10.1007/ s10278-009-9226-4

26. The B (2006) Digital radiographic peroperative planning and postoperative monitoring of total hip replacements-techniques, validation and implementation. Doctoral dissertations, University Medical Center Groningen, The Netherlands

27. Gao X-S, Hou X-R, Tang J, Cheng H-F (2003) Complete solution classification for the perspective-three-point problem. IEEE T Pattern Analysis Mach Intell 25:930-943

28. Wunsch P, Hirzinger G (1996) Registration of CAD-models to image by iterative inverse perspective matching. In: Proceedings of the 1996 international conference on pattern recognition (ICPR 1996), pp 78-83
29. Jonić S, Thévenaz P, Zheng G, Nolte L-P, Unser M (2006) An optimized spline-based registration of a 3D CT to a set of C-Arm Images. Int J Biomed Imaging, pp 1-12, doi:10.1155/IJBI/2006/ 47197

30. Zheng G (2008) Effective incorporation of spatial information in a mutual information based 3D-2D registration of a CT volume to $\mathrm{X}$-ray images. In: Proceedings of the 11th international conference on medical image computing and computer-assisted intervention (MICCAI 2008), Part II, LNCS 5242, pp 922-929

31. Veldpaus FE, Woltring HJ, Dortmans LJ (1988) A least-squares algorithm for the equiform transformation from spatial marker co-cordinates. J Biomech 21(1):45-54

32. Murray DW (1993) The definition and measurement of acetabular orientation. J Bone Joint Surg Br 75(B):228-232

33. Zheng G, Marx A, Langlotz U, Widmer K-H, Buttaro M, Nolte L-P (2002) A hybrid CT-free navigation system for total hip arthroplasty. Comput Aided Surg 7:129-145

34. Lin F, Lim D, Wixson RL, Milos S, Hendrix RW, Makhsous M (2008) Validation of a computer navigation system and a CT method for determination of the orientation of implanted acetabular cup in total hip arthroplasty: a cadaver study. Clin Biomech 23:1004-1011

35. Beckmann J, Lüring C, Tingart M, Anders S, Grifka J, Köck FX (2009) Cup positioning in THA: current status and pitfalls, A systematic evaluation of the literature. Arch Othop Trauma Surg 129:863-872

36. Steppacher S, Moritz T, Zheng G, Zhang X, Kowal J, Suzanne A, Siebenrock KA, Murphy SB (2009) Validation of a new method for determination of cup orientation in THA. J Orthop Res 27:15831588

37. Wan Z, Malik A, Jaramaz B, Chao L, Dorr LD (2009) Imaging and navigation measurement of acetabular component position in THA. Clin Orthop Relat Res 467:32-42

38. Babisch JW, Layher F, Amiot L-P (2008) The rationale for tiltadjusted cup navigation. Bone Joint Surg Am 90:357-365

39. Zhu J, Wan Z, Dorr LD (2009) Quantification of pelvic tilt in total hip arthroplasty. Clin Orthop Relat Res, in press. doi:10.1007/ s11999-009-1064-7, [Epub ahead of print]

40. Dorr LD, Hishiki Y, Wan Z, Newton D, Yun A (2005) Development of imageless computer navigation for acetabular component position in total hip replacement. Iowa Orthop J 25:1-9

41. Dorr LD, Malik A, Wan Z, Long WT, Harris M (2007) Precision and bias of imageless computer navigation and surgeon estimates for acetabular component position. Clin Orthop Relat Res 465:9299

42. Lembeck B, Mueller O, Reize P, Wuelker N (2005) Pelvic tilt makes acetabular cup navigation inaccurate. Acta Orthop 76:517523

43. Dorr LD, Malik A, Dastane M, Wan Z (2009) Combined anteversion technique for total hip arthroplasty. Clin Orthop Relat Res 467:119-127

44. Zheng G (2009) Statistically deformable 2D/3D registration for accurate determination of post-operative cup orientation from single standard X-ray radiograph. In: The proceedings of the 12th international conference on medical image computing and computer-assisted intervention (MICCAI 2009), pp 820-827, Springer 